nahme dieses gefährlichsten, teilweise lebensbedrohlichen Ereignisses bei Myasthenie hat sich in Studien die rasche Entfernung von Acetylcholinrezeptor-Antikörpern gezeigt. Das gelingt sehr gut mittels Plasmaaustausch (PE) oder Immunadsorption (IA). Die bislang fehlenden kontrollierten Daten zur klinischen Sicherheit und Effektivität der Verfahren liefert nun eine 2011 veröffentlichte Studie [2]. Eingeschlossen waren 19 Patienten mit myasthener Krise. Zehn erhielten einen $\mathrm{PE}$, neun zusätzlich zu weiteren gängigen Therapiemaßnahmen eine IA (im Schnitt 3,5 PE sowie 3,4 IA). Beide Methoden senkten laut Reichmann den mittels Myasthenie-spezifischer Skalen erhobenen Myasthenie-Score bis zwei Wochen nach Therapiebeginn ähnlich stark und führten zu einem stabilen klinischen Status (Oosterhuis-Klassen I und II). Allerdings wurde die IA mit zehn versus 16 Nebenwirkungen (eine versus sieben schwere Nebenwirkungen) wesentlich besser vertragen. Angesichts der deutlich höheren Kosten der IA dürfte der PE jedoch weiter indiziert bleiben, schloss Reichmann pragmatisch.

\section{MuSK-positive Myasthenia gravis}

Hohe Relevanz hat nach Ansicht von Reichmann eine Studie, die die Wirk- samkeit einer Therapie mit Rituximab bei Patienten mit MuSK-positiver Myasthenia gravis untersucht hat [3]. Bei der großen Mehrzahl der Myastheniepatienten (ca. 85\%) vermitteln Autoantikörper gegen den Acetylcholinrezeptor (AChR) die Symptome an der motorischen Endplatte der quergestreiften Muskulatur. Nur bei etwa 1-10\% werden Autoantikörper gegen die muskelspezifische Tyrosinkinase (MuSK) nachgewiesen. Diese Patienten sprechen schlechter oder gar nicht auf die herkömmliche Therapie mittels Kortikoiden, Acetylcholinesterase-Hemmern oder milden Immunsuppressiva an.

Dass Rituximab als monoklonaler Antikörper gegen das CD20-Antigen in BZellen eine effektive Therapieoption eröffnet, belegen die Ergebnisse der genannten Langzeitstudie mit einer Beobachtung über fast drei Jahre. Zunächst erhielten 17 Patienten (sechs mit MuSKund elf mit AChR-AK-assoziierter Myasthenia gravis) Rituximab. Es wurde in der Standarddosis von $375 \mathrm{mg}$ pro $\mathrm{m}^{2}$ wöchentlich konsekutiv viermal und dann monatlich für die nächsten zwei Monate appliziert. Bei der Untersuchung nach 31 Monaten waren alle Patienten mit MuSK-AK-assoziierter Myasthenie in Vollremission (4/6) oder hatten nur noch leichte Symptome (2/6). Keiner be- nötigte eine Reinfusion - im Unterschied zu Patienten mit AChR-AK-assoziierter Myasthenie (6/11). Das gute Ansprechen erlaubte eine signifikante $\mathrm{Re}$ duktion der Prednison-Dosis und das Absetzen weiterer Immunsuppressiva, berichtete Reichmann.

Bei der Abschlussuntersuchung wurden neben der klinischen Verbesserung bei der Hälfte der Patienten mit MuSKassoziierter Myasthenie keine MuSKAntikörper mehr gefunden, die andere Hälfte wies eine Reduktion um $80 \%$ auf. Die Therapie verbesserte somit nicht nur den klinischen, sondern auch den immunologischen Status der Patienten. Laut Reichmann Grund genug, Patienten mit MuSK-assoziierter Erkrankung und schlechtem Ansprechen auf konservative Therapien frühzeitig mit Rituximab zu behandeln. Ute Ayazpoor

1. Heckmann JM et al., BMC Neurology 2011; 11: 97

2. Köhler W et al., J Clin Apheresis 2011; 26: 347-55

3. Diaz-Manera J et al., Neurology 2012; 78 : 189-93

NeuroUpdate, Mainz, 16.2.2013

Vortrag "Neuomuskuläre Erkrankungen", Prof. Dr. Heinz Reichmann, Dresden

\title{
Multiple Sklerose: Neues Autoantikörper-Target identifiziert
}

\section{Die lange Suche nach den Schlüsselmolekülen der immunologischen Reaktivität bei der Multiplen Sklerose hat einen Erfolg zu vermelden: Forscher identifizierten einen ATP-sensitiven Kaliumkanal speziell von Gliazellen als immunogenes Ziel von IgG-Antikörpern.}

Seit längerem wird auf der Basis unergiebiger Daten diskutiert, dass Kaliumkanäle eine Rolle als immunogene Targets bei der Multiplen Sklerose (MS) spielen könnten. Die lange Suche nach den Schlüsselmolekülen der immunologischen Reaktivität bei der MS - die laut Professor Volker Limmroth, Klinik für Neurologie und Palliativmedizin, Kliniken der Stadt Köln, bisher vor allem unter den Proteinen und Peptiden des Myelons vermutet wurden - hat nun vielleicht zum entscheidenden Durchbruch geführt: Bei der Untersuchung des SerumIgG von MS-Patienten wurden Antikör- per gefunden, die spezifisch an Gliazellen banden. Im Rahmen eines proteomischen Verfahrens identifizierten die Forscher den ATP-sensitiven, spannungseinwärts gerichteten Kaliumkanal KIR4.1 als immunogenes Ziel von IgGAntikörpern [1]. Beim Vergleich zwischen gesunden Kontrollen, MS-Patienten sowie Patienten mit anderen neurologischen Erkrankungen konnten sie bei Patienten mit MS signifikant häufiger und hochtitriger Serumantikörper gegen KIR4.1 nachweisen, die an die erste extrazelluläre Schleife des KIR4.1 binden. Tierexperimentell wurden nach Injekti- on von KIR4.1-Serum- IgG in die Cisterna magna von Mäusen deutlich weniger KIR4.1-Kanäle in glialen Fibrillen und Astrozyten exprimiert und im Kleinhirn in Arealen mit KIR4.1-Expression eine Komplementkaskade aktiviert. Zumindest in einer Untergruppe von MS-Patienten sehen die Autoren den Ionenkanal als entscheidendes immunologisches Ziel der Autoantikörperantwort. „Sollte sich die Hypothese bestätigen, wäre die Blockade der Antikörper die Basis einer neuen therapeutischen Strategie bei MS“, schloss Limmroth.

Ute Ayazpoor

1. Srivastava R et al., N Engl J Med 2012; 367 : $115-23$

Neuro Update, Mainz, 16.2.2013

Vortrag "MS/Neuroimmunologie",

Prof. Dr. Volker Limmroth, Köln 